\title{
From the fatigue properties of Ti6Al4V produced by ALM selective laser melting process to the mechanical design of an aeronautical part
}

\author{
François Edy ${ }^{* 1}$, Viet-Duc LE ${ }^{2}$, Claudia BIERE ${ }^{3}$, Monica Perez ${ }^{3}$, Etienne Pessard ${ }^{2}$, Franck Morel ${ }^{2}$ \\ ${ }^{1}$ IRT Jules Verne, Bouguenais, ${ }^{2}$ LAMPA, Arts et métiers ParisTech, Angers, ${ }^{3}$ DAHER, Tarbes,
}

\begin{abstract}
$\underline{\text { Abstract }}$
Selective laser melting SLM is investigated through a study of redesign and characterization of an aeronautic part made of titanium Ti6Al4V. The part must ensure an excellent static and fatigue behaviour. The methodology developed hereby follows 3 main steps: First, the influence of laser power, laser speed and hatch distance on the amount/rate of porosity is performed to define optimized process parameters. Then, the influence of building process strategy, i.e. building direction or as-built surface roughness on the static and fatigue behaviour are studied and understood by following a vast experimental campaign. Obtained properties are finally used in a topology optimization study to find the best compromise between part weight and fatigue behavior . 3 prototypes of simulated part are produced and then characterized. Fatigue tests are conducted on the component and confirm the fatigue design proposed. Obtained results are encouraging and illustrate the fatigue design optimization of a complex Additive Manufacturing component.
\end{abstract}

\section{Introduction}

Additive layer manufacturing processes like Selective Laser Melting (SLM) enable the fabrication of highly precise and complex component geometries that are otherwise difficult, costly, or even impossible to realize using conventional techniques [1]. These capabilities are seen by many manufacturer as a way to improve part functionality, to reduce weight and emission for transport, to optimize supply chain and many others modern challenges. However materials produced by this technique often show properties rather different than the ones obtained with conventional manufacturing processes. Thus, to be able to design parts taking into account all the capabilities of this process, more information and additional material databases are required. The French project FATAL (Fabrication Additive Titane Aluminium) managed by IRT Jules Verne has for main objectives to better understand process parameter impact on material properties, to generate a database for industrial designers and correlate this data base with industrial use cases.

\section{Methods and results}

\subsection{Material}

The material studied is Ti6Al4V grade 23 ELI, obtained by inert gas atomization resulting in spherical particles. The particle size distribution was measured by a Mastersizer Malvern laser-based particle size analyzer. 


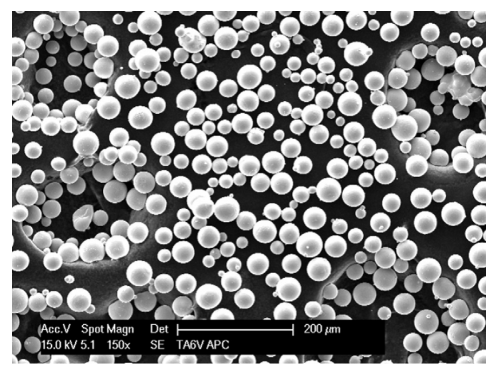

Figure 1 : SEM Analysis of powder particles

The powder batch have the following particle size distribution D10 $=30 \mu \mathrm{m}, \mathrm{D} 50=43 \mu \mathrm{m}, \mathrm{D} 90=61 \mu \mathrm{m}$. Morphology was investigated to define sphericity of particles through shape factor which is the ratio between width and length of particles, the more this ratio is close to 1 , the more spherical the particles are. For this batch, the distribution is the following D10 $=0.73$; D50 $=0.97$; D $90=0.99$ in other words $10 \%$ of the volume of particle has a factor below 0.73 and $50 \%$ has a factor superior to 0.97 , meaning the sphericity of this powder is very good. These analyses were completed by a SEM (Figure 1) which shows that particles are mostly spherical and without satellite.

\subsection{Optimization of SLM process}

To understand the effects of the main parameters on part density, a Design Of Experiment (DOE) was performed. Three parameters were studied in this DOE (laser power P (W), laser speed V $(\mathrm{mm} / \mathrm{s})$, hatch distance $\mathrm{Hd}(\mathrm{mm})$ ), tested values are summarized in Table 1. These values cover a restricted range of energy density Ev from 44.5 to $88.9 \mathrm{~J} / \mathrm{mm}^{3}$.

\begin{tabular}{|c|c|c|c|c|}
\hline Sample designation & $\mathrm{P}(\mathrm{W})$ & $\mathrm{V}(\mathrm{mm} / \mathrm{s})$ & $\mathrm{Hd}(\mathrm{mm})$ & $\mathrm{Ev}(\mathrm{J} / \mathrm{mm} 3)$ \\
\hline 1 & 150 & 750 & 0.1 & 66.67 \\
\hline 2 & 150 & 750 & 0.14 & 47.62 \\
\hline 3 & 150 & 800 & 0.1 & 62.50 \\
\hline 4 & 150 & 800 & 0.14 & 44.64 \\
\hline 5 & 200 & 750 & 0.1 & 88.89 \\
\hline & & & & \\
\hline
\end{tabular}




\begin{tabular}{|c|c|c|c|c|}
6 & 200 & 750 & 0.14 & 63.49 \\
\hline 7 & 200 & 800 & 0.1 & 83.33 \\
\hline 8 & 200 & 800 & 0.14 & 59.52 \\
\hline Ref & 175 & 775 & 0.12 & 62.72 \\
\hline
\end{tabular}

Table 1 : DOE parameter combination and corresponding energy density

On this basis, a complete factorial plan was defined composed of 8 combinations and 3 repetitions, completed with a reference combination. For the optimization program more than 27 cubes $\left(15 \times 15 \times 15 \mathrm{~mm}^{3}\right)$ were produced with parameters reported in the Table 1 .

The layer thickness was constantly $30 \mu \mathrm{m}$, laser beam size was approx. $80 \mu \mathrm{m}$ and substrate plate made of Ti6 Al4V was heated at $200^{\circ} \mathrm{C}$. No post treatment was performed on these parts.

All these cube samples were analysed by optical microscopy and porosity rate in each cube was recorded Figure 2, data from other trials made in the same way with higher energy are added to this figure. This result shows that, in this range of energy, the worst porosity rate is $0,14 \%$ with the lower energy input, the best porosity rate is reached by energy input higher than $60 \mathrm{~J} / \mathrm{mm} 3$ with around $0,01 \%$ of porosity. All these data were introduced in a full factorial plan and analysed with the software Minitab. Obtained model has a $\mathrm{R}^{2}$ of $89.96 \%$ which means that it is well adjusted and a $\mathrm{P}$ value $<0.05$ which means that factor effect is significant. Minitab analyzis identifies the main influent parameters as (by order of importance): Power, interaction Power/ hatch distance and hatch distance.

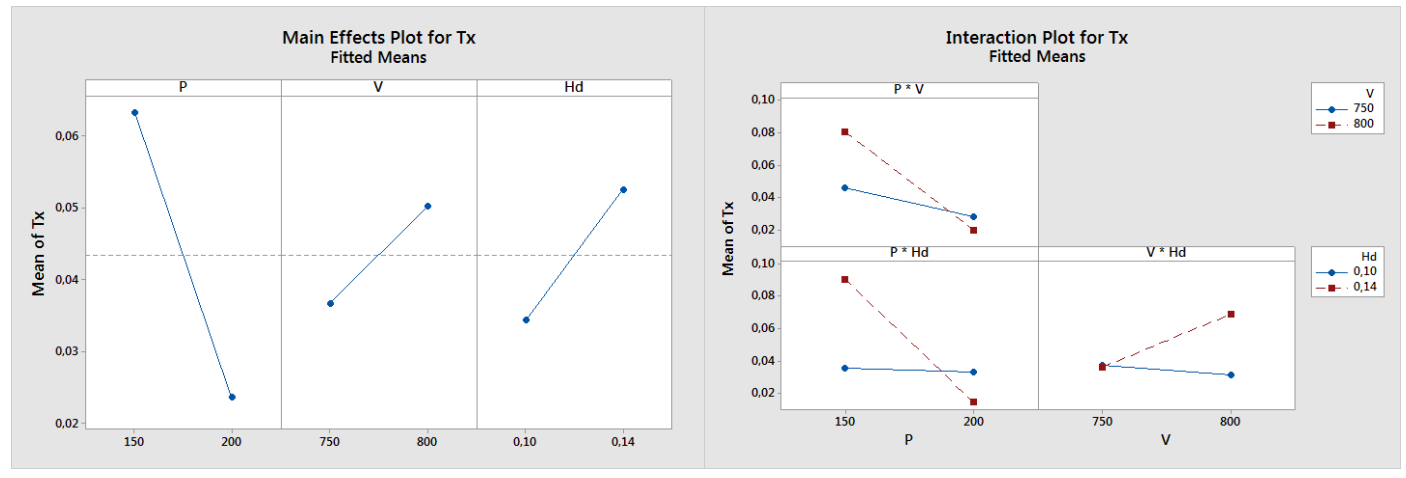

Figure 2 : a) Main effect and b) interaction plot on porosity rate (Tx) 
Figure 2 a) shows that an increasing of laser power decreases the porosity amount, when an increasing of the speed or of the hatch distance increase porosity rate. Interaction plots (Figure $2 b$ ) shows that the lower porosity rate is obtained with the following combinations : high power and low speed, high power and low Hd, low speed and low hatch distance. DOE shows significant effect of factors that are correlated with physical expectation.

The model derived by DOE is written as:

$\mathrm{Tx}=-1,406+0,01696 \mathrm{P}+0,000980 \mathrm{~V}-7,95 \mathrm{Hd}-0,000017 \mathrm{P} * \mathrm{~V}-0,03683 \mathrm{P} * \mathrm{Hd}+0,01917 \mathrm{~V} * \mathrm{Hd}$

With this model we obtain two combinations allowing minimizing porosity rate in this range of value:

\begin{tabular}{|c|c|c|c|c|c|c|}
\hline Parameter set & $\mathrm{P}(\mathrm{W})$ & $\mathrm{V}(\mathrm{mm} / \mathrm{s})$ & $\mathrm{Hd}(\mathrm{mm})$ & $\mathrm{Ev}(\mathrm{J} / \mathrm{mm} 3)$ & Average porosity(\%) & Deviation(\%) \\
\hline 1 & 200 & 750 & 0.14 & 63.49 & 0.0180 & 0.0157 \\
\hline 2 & 200 & 800 & 0.1 & 83.33 & 0.0108 & 0.0108 \\
\hline
\end{tabular}

Table 2: Optimized parameters and average porosity rate obtained

These two solutions were tested one more time with $2 \times 5$ cubes in one batch. Mean porosity rate obtained with the first combination is $0,018 \%$ with a deviation of $0,016 \%$ and for the second one we obtain lower values with an average porosity of $0,011 \%$ with $0,011 \%$ of deviation, as shown in Table 2. These porosity rates are in accordance with literature results obtained on the same material [4].

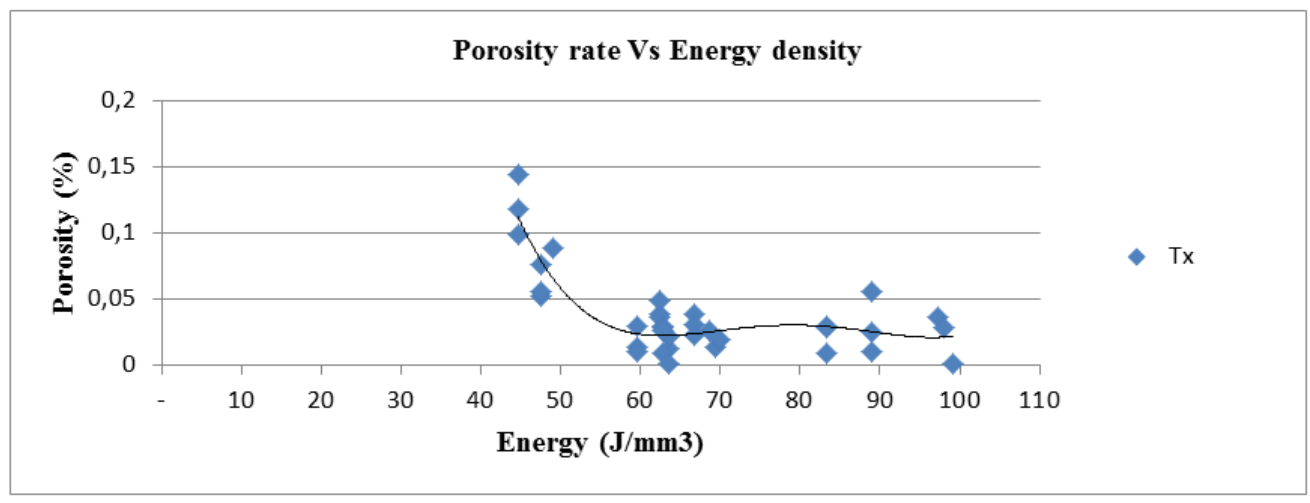

Figure 3: Porosity rate depending on energy density 
Even if the second parameter set with higher energy input offers better results, an energy input of $62.72 \mathrm{~J} / \mathrm{mm} 3$ was used for the rest of the study . Figure 3 shows that an energy increase beyond $60 \mathrm{~J} / \mathrm{mm}^{3}$ does not seem to change the porosity rate, and higher energy density can lead to undesirable effect such as an increase of internal stresses and modification of chemical composition. Recent work [6] also shows that excessive laser powers, combined with large build angles, favor sticking of surrounding particles on overhanging parts in the powder, which degrades the roughness.

\section{3 Fatigue testing}

A vast experimental campaign in fatigue was conducted using cylindrical geometry from ASTM E466 standard (diameter equal to $8 \mathrm{~mm}$ with a gauge length of $25 \mathrm{~mm}$ ). Parts were made using different build orientations $0^{\circ}$ (horizontally), $45^{\circ}$ and $90^{\circ}$ (Vertically). This paper only shows results for $45^{\circ}$ and $90^{\circ}$ as built specimens (Figure 5), as all the $0^{\circ}$ specimens were machined. 3 specimen as-build batches are investigated in this study, referred to as $45^{\circ}$-Batch1, $45^{\circ}$-Batch 2 , and $90^{\circ}$ Batch. Figure 4 shows the $90^{\circ}$ and $45^{\circ}$ fatigue specimen batches.

The surface roughness was scanned with a 3D BRUKER type profilometer. The surface roughness Sa is homogenous around the $90^{\circ}$ surface specimen. For the $45^{\circ}$ specimen the Sa is higher for the downskin. The maximal surface roughness is higher for specimen built at $45^{\circ}$ compared to specimen at $90^{\circ}\left(\operatorname{Sa} \max \left(45^{\circ}\right)=17 \mu \mathrm{m}>\operatorname{Sa} \max \left(90^{\circ}\right)=11 \mu \mathrm{m}\right)($ Figure 4$)$.

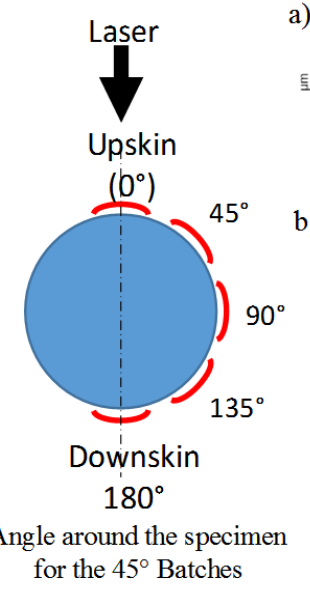

for the $45^{\circ}$ Batches

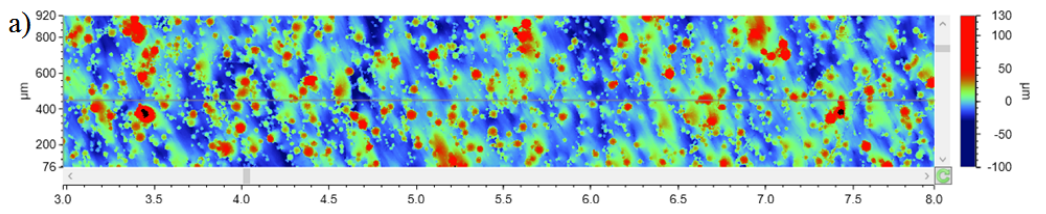

b)

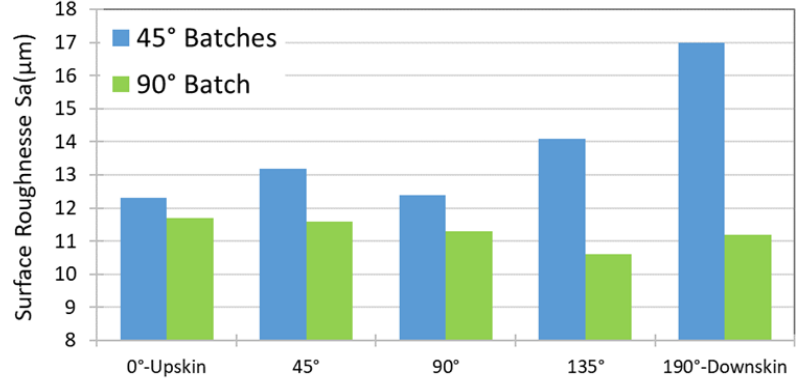

Angle around the specimen

Figure 4:a) example of surface roughness measurement using a 3D profilometer, b) Sa evolution in function of the angle around the specimen

All of the fatigue tests presented below were carried out at ambient temperature under a constant stress amplitude with a loading ratio $\mathrm{R}=0.1$ at a frequency of $20 \mathrm{~Hz}$. A maximum fatigue life of $2 \times 10^{6}$ cycles was used. The stopping criterion was chosen to be the complete failure of the specimen. The run-out specimens that survived $2 \times 10^{6}$ cycles were re-tested at a higher load. All of the fatigue failure surfaces were analyzed on a ZEISS Gemini SEM. 

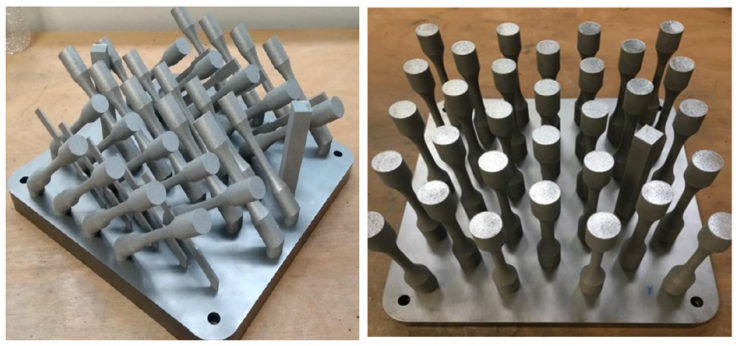

Figure 5:(Left) $45^{\circ}-\mathrm{B} 1$ batch and (Right) $90^{\circ}$ Batch

Figure 6 shows the S-N curves for the 3 investigated batches. The values of the maximum stress, $\sigma_{\max }(1)$, are normalized by the average value of the ultimate strength, $\sigma_{\mathrm{UTS}}$, for the three building directions as defined by the following equation. Note that the maximum difference in the ultimate tensile strength between the three building directions is lower than $5 \%$.

$$
\sigma_{\max , n}=\frac{\sigma_{\max }}{\left(\sigma_{U T S, 0^{\circ}}+\sigma_{U T S, 45^{\circ}}+\sigma_{U T S, 90^{\circ}}\right) / 3}
$$

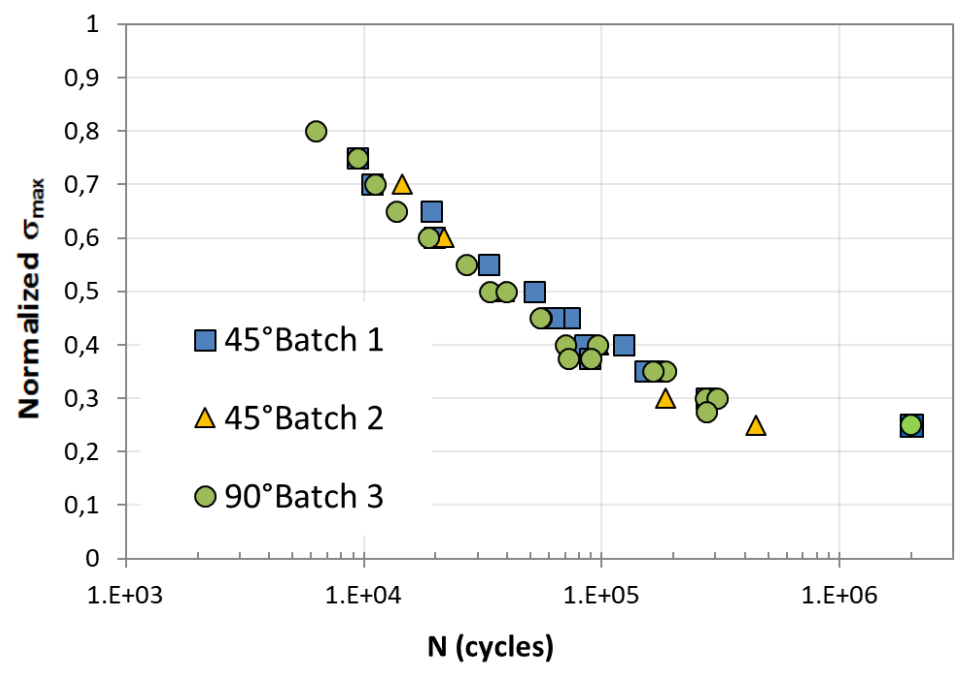

Figure 6:S-N curves obtained on the three different batches

A good repeatability can be observed between the three different batches. The fatigue behavior is therefore isotropic and does not depend on the fabrication direction despite the roughness difference. This result is really different to previous results obtained on machined specimens where a large scatter and some anisotropy are observed [5]. 


\section{4 Crack initiation mechanisms}

Concerning the fatigue damage mechanism, two different kinds of initiation sites could be observed. Initiation started from notch induced by the particular surface topology Figure 7 a); or from surface defect induced by Lack Of Fusion (LOF) Figure 7 b).

For the $90^{\circ}$ batch, the location of the initiation site Figure 7 a) is randomly localized around the specimen.

For the $45^{\circ}$ batch, for all fatigue tests broken after $10^{5}$ cycles, initiation site is localized on the upskin side even if the surface roughness is lower. The presence of surface LOF defect systematically observed at the initiation site after $10^{5}$ cycles control the fatigue behavior. We can therefore assume that LOF defects are mostly present on the upskin side.

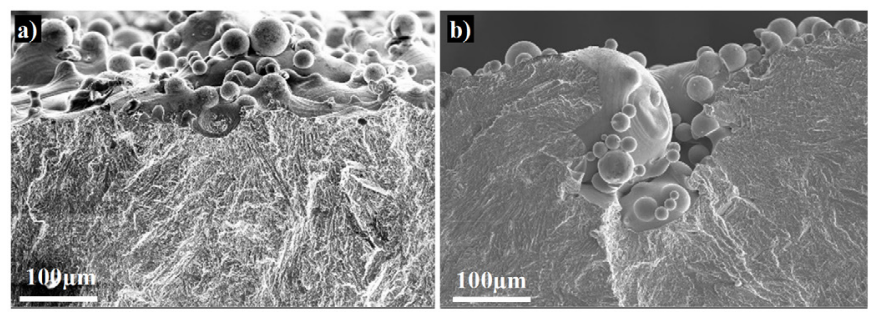

Figure 7: SEM observation of the fracture surface initiation from a) surface notch b) surface defect (Lack Of Fusion)

\subsection{Part design and simulation}

A topological optimisation and finite element analysis was realised on TBM Daher Aircraft movable step to obtain the best design using static and fatigue behaviours as input data compatible with the SLM process. The software used for these analyses was Hyperworks with its different features Inspire, Hypermesh and Optistruct. Requirements taken into account the movable step optimisation were the following ones:

- space and kinematic constraints (increased compactness and compatible use of the aircraft environment around the part),

- the weight (limitation of the mass),

- plane interfaces (no other modification in the aircraft environment),

- mechanical strength (compatible to the loading cases).

Topological analysis leads to a part for which it is necessary to adapt the geometry. Stress and design know-hows are necessary to analyse the results and design finalised part after topological optimisation. Different iterations are necessary to obtain the final part design (Figure 8). 

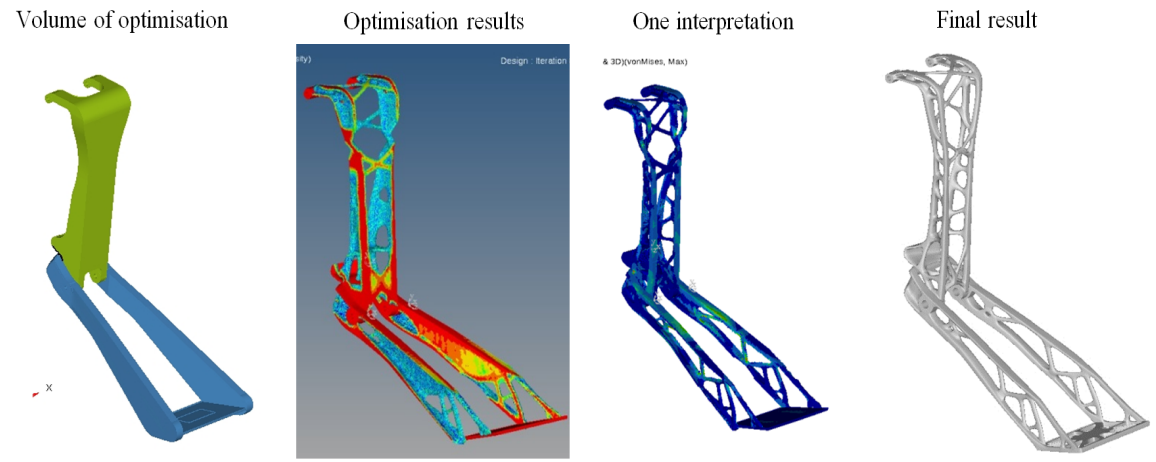

Figure 8 : Optimisation analysis - Part evolution.

Structural justification of aeronautical parts requires input data concerning the material. During the project, tensile tests on elementary specimens were also carried out to supply the FEM analysis for topological optimisation. Mechanical test results lead to the definition of allowable stress in static and in fatigue (Figure 6) used for the movable step design.

The FEM analysis leads to the following results under the fatigue loads (Figure 9a). The maximal principal stress under fatigue load is observed at the top of the movable step. It can be noted that the most loaded areas under static loads are similar to the ones obtained for fatigue loads (Figure $9 \mathrm{~b}$ )).

Safety margins reached with this design are close to zero at ultimate load. This design does not present a problem for the Limit Load. Indeed, the yield strength is not achieved at limit load.

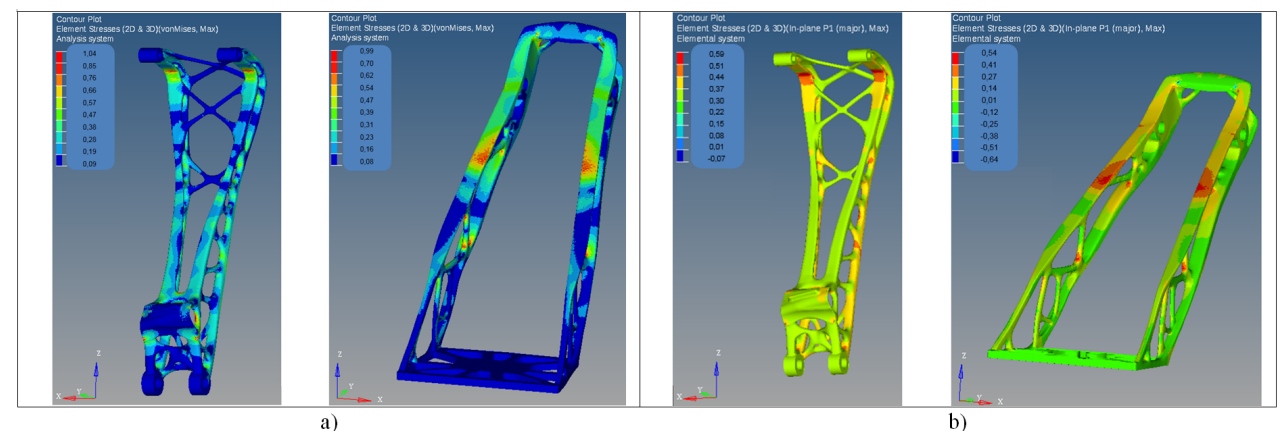

Figure 9 : a) Max Von Mises stress normalized for the static case at UL. b) Max principal stress normalized for the fatigue case.

The max principal stress reached for the fatigue case on the movable step coincides with the point defined on Figure 11 which resulted in a rupture after approximately 20,000 cycles. 
Preparation of manufacturing was performed using Magics19.02 software which allows defining the best orientation and generating support structures. Part orientation was chosen to be a compromise between machine capacities, parts feature, support structure and machining access. Supports are a mix of fully dense and lattice structure to anchor parts to the buildplate and maintain overhanging features. Three normalized fatigue specimens were built on each batch to compare with the first fatigue campaign. Figure 10 shows as built parts and their arrangement on the buildplate. Three batches were produced to get an overview of the repeatability. Second and third builds were the same, while the first job has a different support geometry than the other builds. Indeed this support was too thin and needed to be strengthened. The amount of support for these parts is quite important and it can be divided at least by a factor of 2 by optimising part design and with return of experiment based on first production. This shows the importance of improving the robustness of ALM part manufacturing by enhancing machine and part preparation (CAD/CAM, Simulation). Another way to reduce the amount of support is to use bigger machine to produce parts horizontally.

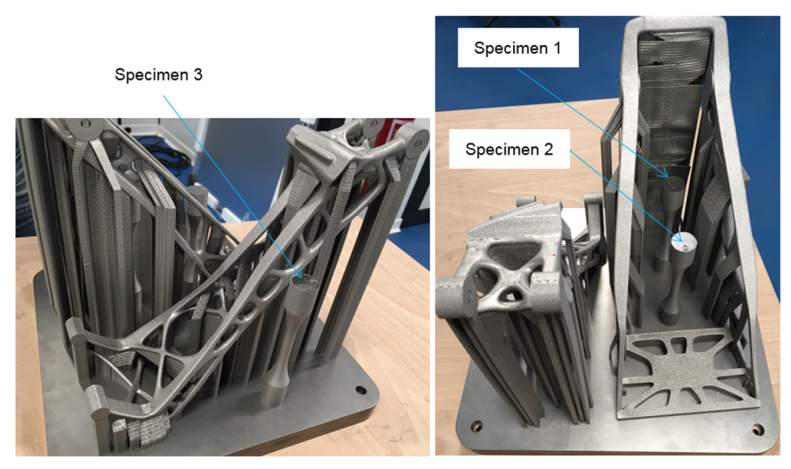

Figure 10 : Batch Movable step/Elementary fatigue specimens.

The produced batches were heat treated on the buildplate after completion in order to remove the internal stresses and then sandblasted to improve the aesthetics and remove the loose powder. Supports were removed manually and critical surfaces machined to obtain expected tolerances.

\section{7) Static and Fatigue part characterization correlation with specimen test}

To correlate result from simulation and real parts, static and fatigue testing were performed. 


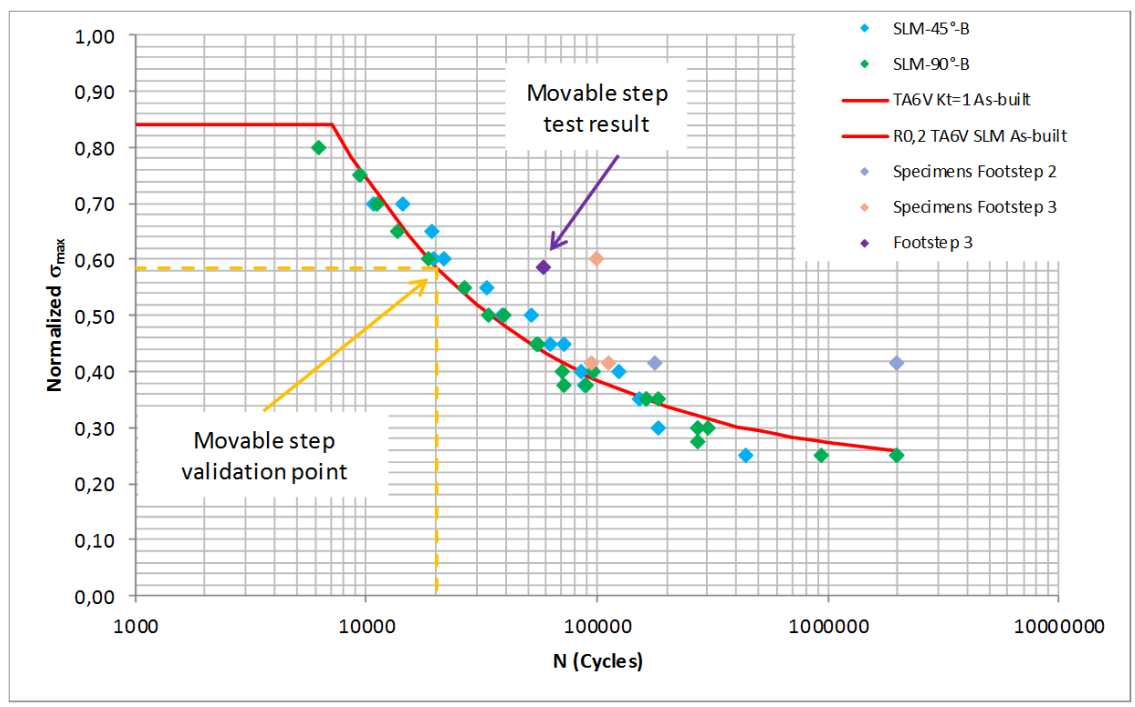

Figure 11 : S-N curve used for the designing : Part characterization correlation with specimen fatigue test.

Static predictions give very good results on most of the strain gauges during the Limit Load and Ultimate load tests. Differences between predictions and tests are less than $10 \%$ on the most constraint gauges at ultimate load with conservative predictions. Due to the high static resistance of the component, the test reaches the limit on displacement and the movable step could not be broken. Static test results are therefore far beyond expectations.

Figure 11 shows the S-N data obtained on as-built $45^{\circ}$ and $90^{\circ}$ samples and resistance limits obtained on the movable step and associated samples. It can be seen that the footstep's specimens have a greater resistance than that of the characterization campaign. This can be explained by the fact that the specimens and the movable steps are sandblasted contrary to test specimens used to obtain the Wöhler curve. A comparison between as built specimens and sandblasted specimens shows sandblasting improve fatigue performance, but also increase fatigue scatter. [2] describes similar results with shot peened specimen and explains this point by the fact that the porosity played a critical role in early crack initiation. Shot-peening improves the surface roughness of parts and induces surface compressive residual stresses balanced by core tensile stresses.

The additive SLM manufacturing process generates defects at the core (pore, un-melted powder, etc.). The presence of residual core tensile stresses adding to the applied stresses causes the specimens break more or less prematurely depending on the occurrence or not of defects in the core of the specimen, which generates this dispersion of results in fatigue [2].

The movable step failure take place at the location predicted by the FEM at the top of the movable step Figure 12. 


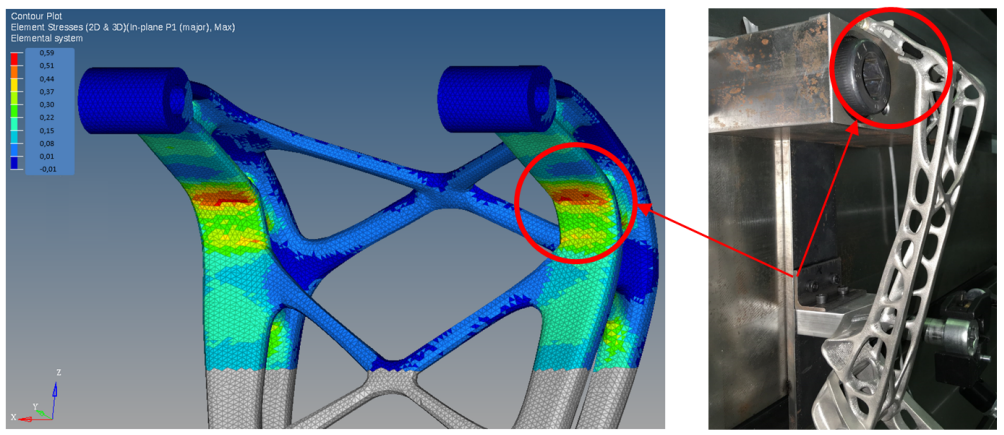

Figure 12 : Max principal stress for the fatigue case / Movable step failure under fatigue loading.

Fatigue predictions using the Wöhler curve obtained on small specimens resulted in a rupture after approximately 20,000 cycles. The failure during the test on the movable step appeared beyond 50,000 cycles ( Figure 12). These results are consistent with numerical analysis expectations and results obtained on fatigue sand blasted elementary specimens. Higher fatigue lives of the components compared to specimens is observed in other works in the literature [3] and could probably be explained by the scale effect. The loaded zone on the component is very small compared to the specimen; this zone is therefore probably defect free and capable higher fatigue strength. This first study shows parts could have been optimized even more in the less busy areas.

\section{Conclusion}

This study covers the complete design cycle from the material characterization to the final testing of the part. The first step of parameter optimization and sensitivity analysis allows the best parameters regarding part density to be defined.. This study highlights importance of energy density on part density and a minimal value of energy density ensuring a good cohesion of particles for Ti6 Al4V of $60 \mathrm{~J} / \mathrm{mm}^{3}$. Fatigue study on as built specimens, shows that fatigue behavior is isotropic in $45^{\circ}$ and $90^{\circ}$ orientation and a small dispersion of results. Investigation of crack initiation mechanisms highlight that fatigue behavior is mainly controlled by surface notch or defects LOF. Design optimization based on this fatigue results allows the design of optimised parts, good failure zone prediction and providing a conservative design. Static tests performed on produced parts are far beyond expectation as the part do not fail. Results from fatigue simulations show a slight deviation compared to part testing. These deviations can be explained by differences of surface treament of specimens and parts asbuilt and sandblasted. Another explanation can be the scale factor occurring from the specimen to the part. Finally parts could be optimized, even more, in the less busy areas. This study shows the ability of additive manufacturing to meet aeronautical specifications. However further work is still needed to ensure quality of parts and improving production reproducibility, which will allow a safety factor reduction and a wider range of applications.

\section{$\underline{\text { Acknowledgement }}$}

This study is part of the FATAL project managed by IRT Jules Verne (French Institute in Research and Technology in Advanced Manufacturing Technologies for Composite, Metallic and Hybrid Structures). The authors wish to thanks the industrial and academic partners of this project; Respectively ACB, Arts et Métiers ParisTech, CNRS, DAHER, Ecole Centrale de Nantes, Europe Technologies, FIVES, Renault and University of Nantes. 


\section{$\underline{\text { Keywords }}$}

Additive manufacturing (AM), Selective laser melting (SLM), Ti-6A-4V, Fatigue behaviour, Porosities, Roughness, Topological optimization, Design for additive manufacturing,

\section{$\underline{\text { Reference }}$}

[1] The role and future of the laser technology in the additive manufacturing environment. Levy, G.N - Physics Procedia 5:65-80, 2010

[2] High Cycle Fatigue (HCF) Performance of Ti-6Al-4V Alloy Processed by Selective Laser Melting. Eric Wycisk, Claus Emmelmann, Shafaqat Siddique, Frank Walther - Advanced Materials Research 816-817, 2013

[3] Electron Beam Additive Manufacturing of Titanium Components: Properties and Performance. Edwards, P., A. O'Conner, and M. Ramulu - J. Manuf. Sci. Eng 135(6),061016, 2013

[4] Improvement of fatigue resistance and ductility of TiAl6V4 processed by selective laser melting. Galina Kasperovich - Journal of Materials Processing Technology, 2015

[5] Interpretation of the fatigue anisotropy of additively manufactured TA6V alloys via a fracture mechanics approach. VD Le, E Pessard, F Morel, F Edy - Engineering Fracture Mechanics, 2019

[6] Koutiri, I., Pessard, E.,Peyre, P., Amlou, O., De Terris, T., Influence of SLM process parameters on the surface finish, porosity rate and fatigue behavior of as-built Inconel 625 parts Journal of Materials Processing Technology, 255, pp. 536-546, 2018 